\title{
No todo se pierde en la traducción. Variedades mundiales de cosmopolitismo*
}

\author{
Gerard Delanty \\ University of Sussex \\ g.delanty@sussex.ac.uk
}

\section{Resumen}

Un importante desafío de los muchos que hoy se plantean al pensamiento cosmopolita es el problema de la traducción conceptual y cultural, puesto que el cosmopolitismo puede ser altamente relevante para ciertos desarrollos del pensamiento indio y chino, incluso cuando no se utiliza el mismo término en las fuentes o interpretaciones. Se abordan tres problemas, a saber: las posiciones universalistas versus las contextualistas; el eurocentrismo, y el problema de las traducciones conceptuales y culturales entre el pensamiento occidental y el no occidental. El argumento central es que el pensamiento cosmopolita debe expandirse más allá de su genealogía occidental para incluir otras tradiciones. Sin embargo, la solución no pasa simplemente por identificar aquellas tradiciones culturales alternativas a las occidentales que puedan contener distintos tipos de valores cosmopolitas, sino por encontrar, en estas distintas tradiciones culturales, recursos para una cosmopolítica. De esta forma, el cosmopolitismo crítico busca plantear una alternativa tanto a las posiciones contextualistas fuertes como a las posiciones universalistas fuertes.

Palabras clave: Asia; cosmopolitismo chino; cosmopolitismo; eurocentrismo; historia global; poscolonialismo; traducción cultural; universalismo.

\section{Abstract. Not All is Lost in Translation: World Varieties of Cosmopolitanism}

Of the many challenging issues facing cosmopolitan thought today, a major one is the problem of conceptual and cultural translation, since it is often the case that cosmopolitanism is highly relevant to Indian and Chinese thought, even though the term itself is

* Traducción de Esperança Bielsa.

Este artículo, cuya primera versión fue presentada en el marco del simposio Conexiones Cosmopolitas en la Universidad de Barcelona, 14-15 de noviembre de 2013, ha aparecido en su versión inglesa en la revista Cultural Sociology, 2014, 8 (4): 374-91. Agradecemos a la editorial Sage el permiso para publicar esta traducción (nota de la traductora). 
not used in the sources or in the interpretations. Three problems are addressed, namely universalist versus contextualist positions, Eurocentrism, and the problem of conceptual and cultural translations between western and non-western thought. The central argument is that cosmopolitanism thought needs to expand beyond its western genealogy to include other world traditions. However, the solution is not simply to identify alternative cultural traditions to western ones which might be the carriers of different kinds of cosmopolitan values, but identifying in these different cultural traditions resources for cosmopolitics. In this way, critical cosmopolitanism seeks to find an alternative both to strong contextualist as well as strong universalist positions.

Keywords: Asia; Chinese cosmopolitanism; cosmopolitanism; cultural translation; Eurocentrism; global history; post-colonialism; universalism.

\author{
Sumario \\ Introducción Cosmopolitismo crítico \\ Problemas del universalismo Conclusión \\ Cuestionar el eurocentrismo Referencias bibliográficas
}

Cosmopolitismo poseurocéntrico

\title{
Introducción
}

El concepto de cosmopolitismo ha tenido una suerte paradójica ${ }^{1}$. Aunque es producto del pensamiento europeo clásico, es considerado cada vez más relevante para las experiencias históricas de las culturas no europeas y refleja cierto autocuestionamiento del eurocentrismo. En este sentido, el cosmopolitismo se ha "cosmopolitizado", aunque se haya privilegiado su genealogía occidental limitando así su relevancia global. A la vista de la importancia global de los países asiáticos, es importante que el cosmopolitismo abarque esos países, especialmente la India y China, que tienen, respectivamente, más del 17 y del 19 por ciento de la población mundial. Las posibilidades de un mundo más cosmopolita dependerán en gran parte de lo que ocurra en ellos. Ambos, que son complejos civilizatorios más que países, poseen ricas herencias cosmopolitas que raramente se vinculan al campo del pensamiento cosmopolita. Hasta ahora, el cosmopolitismo se ha debatido principalmente dentro de los lindes del pensamiento occidental o, alternativamente, es considerado como un término apropiado para referirse a una cultura normativa global ${ }^{2}$. Ha llegado la

1. Se presentaron versiones anteriores de este artículo en la Universidad de Barcelona y en el Congreso Anual de la Asociación de Sociología de Hong Kong, que tuvo lugar los días 7 y 8 de diciembre de 2013. Agradezco los comentarios de tres evaluadores de la revista Cultural Sociology y de muchos otros que asistieron a las mencionadas exposiciones.

2. Ha habido algunos intentos de ir más allá del cosmopolitismo occidental, pero la literatura al respecto es limitada y, a menudo, adopta posiciones polémicas alrededor de un cosmopolitismo alternativo no occidental (véase una contribución importante en Breckenridge et al., 2002; véase también Mignolo, 2000b). 
hora de ir más allá del eurocentrismo latente que persiste en el pensamiento cosmopolita. ¿Será posible hacerlo sin pasar de una posición contextualista a otra y sin que algo se pierda en la traducción?

De los muchos desafíos, uno es el problema de la traducción conceptual y cultural, dado que el cosmopolitismo puede ser altamente relevante para ciertos desarrollos del pensamiento indio y chino, incluso cuando no se utilice el mismo término en las fuentes o interpretaciones. Ciertamente, el uso que se hace del término puede esconder aplicaciones más relevantes si se encuentran formas aceptables de traducción conceptual y cultural. Sin embargo, tales empeños también conllevan el peligro de una pérdida de significado, puesto que diferentes conceptos traducen experiencias distintas. También está la compleja cuestión de qué registro de significado debe privilegiarse, los conceptos explicativos occidentales o los no occidentales, o de si existe la posibilidad de encontrar un lenguaje alternativo. Este artículo aborda centralmente este desafío metodológico.

El cosmopolitismo es, principalmente, una idea normativa sobre el mundo que adopta una variedad de formas culturales en las que ésta se expresa. Esto significa que existe una cierta tensión entre la idea y sus formas manifiestas, pues las ideas no son abstractas en su totalidad y, debido a la multiplicidad de formas posibles, es difícil especificar los aspectos que definen al cosmopolitismo. No obstante, no nos ayuda mucho empezar una investigación sin por lo menos precisar algo aunque sea de forma provisional, por lo que intentaré ofrecer una definición mínima de cosmopolitismo antes de considerar las implicaciones de su pluralización y extensión más allá de sus presupuestos históricos y conceptuales occidentales.

Como idea normativa en el sentido más general, el cosmopolitismo otorga relevancia a la perspectiva del otro y a situarse en una totalidad más amplia, que, generalmente, puede referirse al mundo, tal como lo indica el término griego cosmos, que significa la comunidad mundial en oposición a una definición más estrecha de comunidad. Así, el término original kosmopolitês significaba 'ciudadano del mundo'. Más allá de este sentido general, el concepto ha tenido una historia diversa y experimenta en la actualidad un nuevo cambio de significado, que ya no se define exclusivamente por su herencia europea ${ }^{3}$. Ello conlleva el riesgo de que se "pierda en la traducción» (por ejemplo, en lo que se refiere a su importancia normativa y a sus presuposiciones cognitivas), así como también nuevas oportunidades para la innovación conceptual. Sin embargo, la idea central del cosmopolitismo permanece, a saber, la afirmación de que los individuos y los grupos a los que pertenecen tienen obligaciones hacia los otros más allá de su contexto inmediato (Brock, 2009).

Este principio normativo, que Kant asociaba al de la hospitalidad, ha vinculado el cosmopolitismo a la tradición del pensamiento liberal, que afirma cier-

3. No existe un único cosmopolitismo occidental, sino varios, y el concepto ha evolucionado considerablemente a lo largo de su historia; por ejemplo: Kant, Marx y Alexander von Humboldt representan diferentes concepciones de cosmopolitismo. Véase también Kleingeld (1999). 
tos principios universales de justicia que todas las sociedades deben encarnar. Mientras que, por lo general, estos principios se relacionaron históricamente con las sociedades occidentales, cada vez más están en la base de la mayoría de las concepciones y de la reivindicación de los derechos en el mundo entero. Ello otorga al cosmopolitismo un cierto universalismo que no debería confundirse con la dominación occidental, a pesar del hecho de que Europa haya podido reclamarlo como su propia herencia o de que se haya utilizado para justificar iniciativas imperialistas.

El cosmopolitismo es hoy, más bien, una fuente de resistencia contra la dominación occidental. Su ethos universalista se ha desplegado de manera predominante al servicio de aplicaciones particularistas, como en las apropiaciones subalternas o poscoloniales. En este artículo, defiendo la posibilidad de que se realice una traducción conceptual y cultural satisfactoria del cosmopolitismo que permita relacionarlo con tradiciones no occidentales de investigación. Lo que se necesita es una comprensión más intercultural del cosmopolitismo como una tradición intelectual y política relevante en todas las culturas del mundo. Aunque ello no necesariamente produzca un proyecto cosmopolítico, constituirá un paso esencial para cosmopolitanizar el diálogo global y para superar los rastros de eurocentrismo.

Las aproximaciones dominantes al cosmopolitismo en la teoría política aceptan con demasiada facilidad los fundamentos occidentales del término sin tener en cuenta los problemas de la traducción cultural. La contribución específica de este artículo es plantear la cuestión de la traducción cultural para el cosmopolitismo y explorar la posibilidad de que las tradiciones no occidentales puedan contener valores cosmopolitas diferentes a los occidentales. Este enfoque va más allá de una visión del cosmopolitismo como una condición universal o simplemente como diálogo intercultural. Al invocar la dimensión crítica del cosmopolitismo, también se afirma —siguiendo el ensayo de Walter Benjamin de 1923 «La tarea del traductor» (1970) — la lógica de la traducción como un modo de transformación radical y que el mismo cosmopolitismo puede constituir una forma de traducción cultural que crea nuevos modos de apertura al mundo.

\section{Problemas del universalismo}

Hoy, el universalismo no significa que existan creencias universalmente válidas o un orden objetivo de verdades, sino que hay condiciones mínimas que todas las sociedades deben cumplir y reconocer y que pueden hacerse ciertas presuposiciones respecto al valor moral de la persona y a la naturaleza de la justicia. Las exigencias del universalismo varían de expectativas fuertes a débiles dependiendo de si se trata de asuntos culturales, morales o científicos. Estos últimos proporcionan las demandas más fuertes, pero también nos recuerdan, como reconoció Kant, que, mientras la ciencia, el gusto estético y la ley y la moral son muy distintas, la naturaleza del universalismo es, hoy, principalmente justificatoria, tal y como ha mantenido Benhabib (2011: 62-5): las 
afirmaciones deben justificarse y establecerse a través de procedimientos de investigación, evidencia, autorreflexividad e interrogación. En este sentido, no se puede sostener ni el relativismo total ni un universalismo basado en un orden objetivo de verdades. El universalismo cosmopolita no establece valores específicos que las sociedades deban profesar o practicar, sino que exige el reconocimiento de formas de solucionar problemas y de institucionalizar la justicia. Se trata más de un universalismo de principios que de valores y su sustancia es más procedimental que fundacional: las normas cosmopolitas no existen simplemente como verdades atemporales encarnadas en los textos o en las mentes de los pensadores que es necesario descubrir, sino que se crean. Esa noción del universalismo hace tiempo que se ha enterrado.

Contra aquellos ${ }^{4}$ que mantienen una noción de verdad más históricamente contextualizada, la posición justificatoria que defiendo enfatiza no la relatividad de un contexto histórico o de un entorno cultural para la validez normativa, sino los procedimientos y los supuestos que constituyen una parte integral de todas las sociedades en el mundo moderno. Siguiendo a Habermas, afirmamos que pueden hacerse ciertas presuposiciones sobre la naturaleza del individuo y sobre la comunidad política dejando abiertas las soluciones específicas que hay que encontrar a los problemas que se confrontan, puesto que muchos de ellos están ligados a su contexto. A esto, yo añadiría la dimensión de las capacidades cognitivas, que no son simplemente principios abstractos de resolución de problemas, sino habilidades, capacidades de aprendizaje y competencias adquiridas. La dificultad reside en separar las prácticas justificatorias — que pueden tomarse por universales - de las suposiciones culturales (por ejemplo: ¿es una suposición cultural que la gente sea razonable y capaz de autocuestionarse o que todos los individuos tengan un derecho moral a ser respetados?). Se trata de una cuestión muy compleja a la que no es fácil responder en un plano general con una afirmación históricamente justificable. De hecho, existen ejemplos de cuando puede no tratarse de una suposición válida. Sin embargo, considero que es posible afirmar que, en las sociedades modernas, se pueden hacer estas suposiciones y, en consecuencia, tanto los relativistas o contextualistas extremos como los universalistas se equivocan.

Para mí, el cosmopolitismo ofrece una posición intermedia: defiende ciertas capacidades universales en las sociedades humanas para buscar respuestas a ciertas preguntas, reconociendo que la forma en la que se formulan las preguntas y se encuentran soluciones a ellas estará influida —aunque no determinada, como mantendrían los contextualistas- por el contexto cultural, y que los resultados no serán los mismos, como requerirían los universalistas, puesto que son posibles justificaciones y resultados distintos. Por estas razones, el cosmopolitismo es un fenómeno pluralizador al tiempo que mantiene una relación

4. Mientras que la filosofía del siglo xx es, en gran parte, si no en su totalidad, posuniversalista, la ciencia social a menudo se ha quedado rezagada en términos de sus afirmaciones normativas o cognitivas y adopta con frecuencia, implícita o explícitamente, presuposiciones contextualistas fuertes o más universalistas. Para una discusión reciente, véase Lukes (2000). 
con la unidad. La relación entre el contexto cultural (que puede denominarse el contexto de la justificación) y las capacidades cognitivas y los principios normativos a través de los cuales los individuos solucionan los problemas es abierta y altamente contingente, lo cual hace posible la diversidad. La existencia empírica del cosmopolitismo, que es lo que lo hace sociológicamente interesante, reside en esta diversidad de formas posibles. El desafío para el cosmopolitismo sociológico es comprender el proceso a través del cual emerge el cosmopolitismo (véase, por ejemplo, Beck, 2006; Rendall et al., 2009; Skrbis y Woodward, 2013). En otras palabras, se trata de cómo emerge una idea normativa y se encarna en formas sociales. Podemos elegir no denominarlo cosmopolitismo, pero yo lo hago por ahora, pues no existe un término alternativo en el léxico de la ciencia social. No se puede sustituir por globalización, que no es un concepto normativo. En efecto, el amplio atractivo del cosmopolitismo no está desconectado de una crítica a la globalización.

El cosmopolitismo se ha convertido en una parte inevitable de la composición de las sociedades modernas y ejemplifica el espíritu de la modernidad, al articular normas de crítica, reflexividad y autoproblematización. La ética cosmopolita entra en juego en el encuentro de una sociedad o cultura con otra, especialmente a la luz de la necesidad de resolver problemas comunes. No es mi intención en este artículo analizar con más detalle el contenido teórico del cosmopolitismo ${ }^{5}$. El objetivo del presente artículo es considerar las implicaciones de las variedades mundiales de cosmopolitismo y si hay una forma de traducir entre ellas. Para ello, han de considerarse dos problemas relacionados, a saber, el eurocentrismo y el problema de las traducciones conceptuales y culturales. Las primeras dos secciones tratan sobre el eurocentrismo y su crítica. La tercera sección ofrece una breve consideración de lo que denomino cosmopolitismo crítico, que va más allá de la crítica del eurocentrismo para identificar escenarios alternativos a través de actos de traducción cultural.

\section{Cuestionar el eurocentrismo}

En la actualidad, la mayoría de las versiones del cosmopolitismo rechazan el universalismo fuerte y se aproximan en distintos grados a las posiciones contextualistas. Esto refleja la tendencia hacia concepciones culturales del cosmopolitismo y un nuevo interés por el tema dentro de las ciencias sociales, donde hay ahora muchos intentos interesantes de aproximación empírica a lo que era un concepto más bien normativo de la filosofía política. Dos desarrollos relacionados han contribuido en gran medida a ello. Primero, la aplicación del cosmopolitismo al mundo no occidental más amplio, tal y como refleja especialmente la popularidad del concepto en la antropología (véase Werbner, 2008). Segundo, el movimiento general contra el eurocentrismo. La aplicación del cosmopolitismo a las sociedades no occidentales ofrece oportunidades

5. Lo he hecho en otro lugar (Delanty, 2009, 2014; véanse también las contribuciones de Delanty, 2012). Véase también Beck (2006), Cheah (2006) y Holton (2009). 
prometedoras para la ciencia social no eurocéntrica. Efectivamente, en muchas aproximaciones, parecería que el cosmopolitismo es precisamente la producción de interpretaciones no occidentales del mundo (Mignolo, 2000a). Si lo examinamos más detalladamente, es posible identificar por lo menos cuatro posiciones en lo que el cosmopolitismo no eurocéntrico conlleva en términos de sus suposiciones teóricas y metodológicas. Estas se expondrán en la siguiente sección, pero primero ofreceré una breve consideración de lo que se supone que es el eurocentrismo, dado que el problema del eurocentrismo es lo que obliga en un primer momento a encontrar alternativas. Existen también cuatro posibles significados de este término tan confundido y vilipendiado, aunque el primero se puede descartar por ser hoy menos relevante ${ }^{6}$.

Universalismo civilizatorio: la afirmación de que Europa u Occidente son, intelectualmente, moralmente o políticamente, superiores en los cimientos de su civilización al resto del mundo. Se trata de una afirmación normativa de ejemplaridad que hoy raramente se defiende en las ciencias sociales, aunque era corriente en el periodo colonial y ha definido lo que, desde Edward Said, generalmente se considera como el «orientalismo»: la afirmación de que Occidente puede conocer el mundo no occidental en términos definidos por occidente $y$, dado que esos términos son esencialmente relaciones de poder, no existe el Otro como tal, puesto que el Otro es una construcción del dominante. Hacia 1945, este tipo de orientalismo fue, en su mayor parte, reemplazado por lo que denomino universalismo analítico.

Universalismo analítico: la afirmación menor de que los patrones europeos de desarrollo de la sociedad son el modelo básico para comprender el resto del mundo constituye un orientalismo residual más común, atribuible a la mayoría de las teorías de la modernización. Se hallaba en parte implícito en la sociología clásica, por ejemplo, en Weber. Difiere del orientalismo en que no hace presunciones normativas sobre lo que son categorías empíricas. Los errores cometidos acerca del mundo no europeo pueden atribuirse a la mala investigación más que a la creencia en la superioridad moral de Europa. Ésta es una de las formas más difundidas de eurocentrismo que, generalmente, implica privilegiar las formas europeas u occidentales de conocimiento y considerar que el conocimiento no occidental no es científico y necesita ser traducido al discurso occidental. También puede verse como parte de un proceso más amplio de «occidentalización». Hay que puntualizar que no siempre se lo promueve desde Occidente y que son a menudo los intelectuales de tradiciones culturales muy distintas a las occidentales los que lo llevan a cabo. Muchos intelectuales chinos del movimiento Aprendizaje Occidental de mediados del siglo XIx favorecieron las ideas occidentales por encima de las confucianas (véase Jenco, 2012). Otro ejemplo pertinente es el proyecto quemalista de occidentalización de Turquía en el siglo XX.

Excepcionalismo europeo: la afirmación más moderada de excepcionalidad en vez de ejemplaridad mantiene que la ascensión de Europa puede explicarse

6. Para una clasificación distinta de los tipos de eurocentrismo en las ciencias sociales, véase Wallerstein (1997). 
de forma más o menos exclusiva en referencia a procesos endógenos de desarrollo, sin tomar en cuenta la contribución positiva del mundo no occidental o sin considerar el imperialismo donde este fuera relevante. Se trata de una posición que se ha adoptado ampliamente y sobre la que se ha debatido mucho, especialmente desde que Pomeranz (2000) y otros, desde la perspectiva de la historia global, han demostrado la importancia de tener en cuenta que el mundo es un sistema integrado. Así, Conrad (2012) ha mostrado cómo la Ilustración debe situarse en un contexto global y que no se trata únicamente de un fenómeno europeo.

Europeísmo conceptual: la aplicación de conceptos, categorías y teorías de origen europeo al mundo no europeo. Se trata de una forma más débil de eurocentrismo y el objetivo de la crítica que hace Chakrabarty (2000) de las presuposiciones eurocéntricas de la investigación histórica (véanse también las versiones sociológicas de Alatas, 2006; Connell, 2006; Sitas, 2006). En este caso, el problema no es el privilegio explícito de la sociedad europea, como en el universalismo analítico, sino el uso de conceptos que derivan de la historia europea en contextos en los que no pueden aplicarse. El resultado es el privilegio cognitivo y epistemológico de modos de pensamiento occidental.

En referencia al uso del término, existe una quinta posibilidad, completamente peyorativa: la investigación basada en Europa. Sin embargo, considerar que la investigación que sólo se refiere a Europa es eurocéntrica es algo vacío, incluso cuando esta investigación sea de poco interés para los cosmopolitas.

En mi opinión, de estas posiciones, sólo la cuarta constituye un problema significativo para el cosmopolitismo, que es un término que deriva del pensamiento europeo y presenta desafíos de traducción cultural y conceptual. Hay serios problemas de traducción conceptual en cualquier intento de llevar el cosmopolitismo más allá del eurocentrismo. En efecto, la misma palabra griega es ya una indicación del problema. Puede ser necesario encontrar ejemplos de cosmopolitismo que no necesariamente se correspondan con algunos de sus usos en la tradición occidental. El problema que aborda específicamente este artículo es, por lo tanto, si el cosmopolitismo puede escapar a este tipo de eurocentrismo.

Cualquier afirmación de eurocentrismo debe clarificar exactamente a qué versión se opone para evitar el argumento del hombre de paja de un discurso orientalista continuo desde principios del siglo xix hasta hoy. En lo que sigue, se discuten cuatro tendencias del cosmopolitismo poseurocéntrico.

\section{Cosmopolitismo poseurocéntrico}

Aunque gran parte de la teoría cosmopolita no aborda la temática del eurocentrismo y se ocupa más bien de los problemas normativos de la justicia global, el problema del eurocentrismo se ha agudizado debido a la recepción del cosmopolitismo en muchas partes del mundo, especialmente en Asia. La investigación histórica y cultural del cosmopolitismo también ha subrayado la necesidad de que este atienda al eurocentrismo incipiente. A continuación, se 
presentan las principales variedades de cosmopolitismo que suponen un intento de dirigirse a las culturas cosmopolitas del mundo no occidental.

\section{Liberalismo cosmopolita}

Se trata, simplemente, de la extensión del pensamiento liberal, incluyendo el cosmopolitismo europeo, para comprender las contribuciones de otras partes del mundo. Las presuposiciones siguen siendo las del pensamiento europeo, pero mejoradas para incluir las voces de otras tradiciones. Puede ser considerado una continuación del multiculturalismo a nivel global. Se trata, principalmente, de un intento de representar tradiciones culturales no europeas dentro de un sistema de pensamiento creado por Europa. No puede dudarse de que la mayoría de las concepciones del cosmopolitismo adoptan esta forma, que puede verse como una corrección más que una alternativa al cosmopolitismo clásico. Como enfoque antieurocéntrico, se trata de un intento de superar el «eurocentrismo analítico» de las corrientes principales del saber. Puede objetársele que no se toma suficientemente en serio el pensamiento no occidental como generador de conocimiento en sus propios términos, puesto que los modos occidentales de pensamiento poseen una legitimidad previa y dictan, además, las fuentes que pueden tomarse como ejemplos de conocimiento no occidental relevante. La suposición es siempre que los recursos intelectuales de Occidente poseen una relevancia universal y que no pueden conocerse en sus propios términos, tal como afirma Godrej (2011) sobre la filosofía política. Así, el pensamiento no occidental representado por Gandhi, Confucio e Ibn Rushd (Averroes) se valora porque estos pensadores abordan cuestiones que se consideran relevantes para el pensamiento occidental. El resultado, siguiendo a Godrej (2011), es el abandono de otras fuentes que no se tienen por traducibles a las categorías occidentales. Existe, por lo tanto, un problema metodológico sobre qué fuentes seleccionar como representaciones del cosmopolitismo. Una consideración adicional es si el objetivo más amplio es la comprensión cultural o la conmensurabilidad de las tradiciones (véase Jenco, 2007, 2012). Generalmente, la tradición liberal no llega a la conmensurabilidad o a una posibilidad de innovación más allá de un amplio intento de entender al Otro en los términos dictados por el discurso liberal occidental.

\section{Universalismo cultural}

Una variante de la posición anterior es el esfuerzo más humanista por identificar valores comunes entre las culturas, sin presuponer necesariamente una cultura como el punto de referencia. Los presupuestos son liberales en la aspiración de identificar valores universales. Estos enfoques generalmente toman las principales religiones del mundo como fuentes de valores comunes, como la noción musulmana de umna, el concepto hindú de dharma o la noción africana bantú de ubuntu. Esta comprensión del cosmopolitismo 
puede relacionarse con la noción de un «diálogo de civilizaciones» ${ }^{7}$. Cualquier aproximación universalista de este tipo tendrá que conformarse con la identificación de un conjunto mínimo de valores que todas las culturas comparten, por ejemplo: la noción de «humanidad común». Aunque ofrecen correctivos importantes a la mayoría de eurocentrismos, estos enfoques se ven limitados por restringirse a modelos dialógicos de cosmopolitismo basados, en su mayor parte, en interpretaciones de las tradiciones premodernas $y$, frecuentemente, poseen un objetivo religioso, como en el llamamiento del papa Juan Pablo II en 2001 a un diálogo de civilizaciones. También pueden limitarse en términos de los tipos de problemas que se postulan como de interés universal, ignorando temas que pueden tener menos relevancia universal, pero ser de mayor importancia para una religión mundial. Esto tiene que ver con el problema de cómo se identifica en primer lugar el discurso cosmopolita y si es posible traducir conceptos antiguos en términos modernos, así como en registros de significado de otras tradiciones culturales donde los términos pueden no ser reconocibles debido a experiencias históricas distintas en relación con, pongamos por caso, estructuras sociales y patrones de formación del estado. Así, Gray (2010) mantiene que, en el caso de las tradiciones brahmánicas y védicas de la antigua India, muchas interpretaciones incorrectas se apoyan en suposiciones históricas y en categorías explicativas europeas, por ejemplo: en conceptos de la tradición del contrato social del liberalismo occidental, como el estado de naturaleza. Otro grave problema de estos enfoques es la falta de un compromiso crítico con las tradiciones culturales y religiosas en cuestión y, generalmente, se acepta que contienen las semillas de los valores universales del presente. Aunque puede afirmarse que, en Asia, la religión es todavía una fuente importante de la identidad política y cultural, no sucede lo mismo en Europa. El cosmopolitismo europeo tiene muy poco que ver con el cristianismo. Lo mismo sucede con las expresiones latinoamericanas del cosmopolitismo (véase Mota, 2012). Sin embargo, como ha mantenido Fred Dallmayr (1996: XVIII) siguiendo a Heidegger y a Gadamer, el diálogo no necesariamente significa la representación de un consenso previamente existente, sino que puede implicar una orientación hermenéutica hacia una comprensión más profunda de la diferencia. Pero esto requeriría un paso más allá de la identificación del cosmopolitismo con las culturas de las religiones de la era axial y un compromiso más fuerte con la modernidad.

\section{Cosmopolitismo alternativo}

De alcance menos universalista, esta posición implica el intento de encontrar un cosmopolitismo indígena no occidental en las tradiciones no europeas. En este caso, la preocupación es lo particular más que lo universal. Más que

7. En 1999, el expresidente de Irán, Mohammad Jatamí, usó el término como una alternativa a la tesis del choque de civilizaciones. 
buscar la conmensurabilidad o la traducción de lo local a lo universal, el propósito es la particularización de lo universal. Se encuentran ejemplos de cosmopolitismos alternativos en las tradiciones precoloniales o en las culturas que no fueron principalmente conformadas por la influencia de la civilización europea, como China, Japón, Irán y el mundo otomano antes de 1918 o, en el caso de las que lo fueron, como la India, donde su influencia no borró las tradiciones más antiguas. Por ejemplo, Euben (2008) ha demostrado la existencia de un cosmopolitismo islámico arraigado en el viaje y en los mundos culturales móviles (véase también Meijer, 1999). Pollock (2006) mantiene la existencia de una tradición cosmopolita sánscrita diferente pero comparable a la herencia europea basada en la lengua latina, contra la tradicional asociación del cosmopolitismo al universalismo occidental en oposición al particularismo asiático. Puede verse el socialismo internacional como un tipo específico de cosmopolitismo que ha tenido un considerable atractivo en China y la ha vinculado a Rusia durante gran parte del siglo precedente (véase Rofel, 2012). A principios del siglo xx, había formas rivales de cosmopolitismo, una influida por Occidente y otra más interna y nacionalista china (Cheah, 2006; Rofel, 2012). Existe una amplia literatura de estudios comparativos sobre la filosofía europea, china e india que es relevante en este contexto (véase Angle, 2012; Burik, 2009) ${ }^{8}$. Estos enfoques pueden describirse como posliberales, en tanto que implican un cierto relativismo y el reconocimiento de los límites de la conmensurabilidad de la traducción y de la comparación cultural. Son ejemplos importantes del cosmopolitismo alternativo los debates chinos sobre el confucianismo como base para un tipo distinto de democracia, como en las obras de Daniel Bell $(2000,2006)$. La escritura del filósofo confuciano Mencius, que defendía una noción de humanidad común, o el debate sobre el concepto chino de ren (que significa algo así como 'humanidad', pero que también puede traducirse por 'amar a los otros') constituyen otro ejemplo pertinente de un concepto de importancia universal que puede abordarse desde un contexto civilizatorio distinto sin presuponer el pensamiento occidental, como mantiene Ackerly (2005). Sin embargo, estas aproximaciones podrían contener un cosmopolitismo insuficiente, debido a que simplemente identifican caminos alternativos hacia la democracia cuyas presuposiciones son distintas a las de la democracia liberal occidental.

Bajo esta denominación, también podemos incluir las concepciones poscoloniales y subalternas del cosmopolitismo (Go, 2013a, 2013b; Mignolo, 2000a). La crítica poscolonial del eurocentrismo ha sido importante para abrir nuevas preguntas y caminos de investigación, particularmente en referencia a la hibridez. Sin embargo, padece de concepciones unilaterales de la herencia europea que exageran el colonialismo en la constitución de Europa y reducen de manera excesiva las historias no occidentales al impacto de Europa. El resultado sobreestima las tradiciones no occidentales como «alternativas». El poscolonialismo también resulta muy limitante en el caso de las muchas regiones del mundo

8. Véanse numerosas ilustraciones en la revista Philosophy East and West. 
que no sufrieron el imperialismo occidental y que fueron ellas mismas poderes imperiales (por ejemplo: China, Japón, Irán y Turquía), donde el imperialismo fue parcial e incompleto o donde el país colonizado (por ejemplo: Brasil) se convirtió en un poder imperial que aventajaba a su amo europeo.

Una tercera estrategia es la de encontrar geografías alternativas que cuestionan las presuposiciones de la visión recibida de la historia, que se organiza en civilizaciones y continentes separados. Desde la perspectiva de la historia global, opuesta a una visión de la historia centrada en la nación o arraigada en tradiciones civilizatorias supuestamente auténticas, se han enfatizado los encuentros culturales y las regiones transfronterizas. Esto ha conducido a una literatura emergente sobre geografías alternativas y encuentros civilizatorios, gran parte de la cual adopta el cosmopolitismo como marco ${ }^{9}$. Así, el océano Índico se ve cada vez más como un espacio transnacional en el que se conectaban el África Oriental y el Asia Suroriental. Getz (2013: 40) comenta la naturaleza cosmopolita de la sociedad de habla swahili. Construida entre las poblaciones de habla bantú del África Oriental, integraba a somalís, árabes y surasiáticos, y los swahiliparlantes viajaban a la India y a Arabia (véase también Sheriff, 2010; Hawley, 2008). Pueden formularse argumentos similares sobre otras regiones del mundo, por ejemplo: el Asia Central (Liu, 2011) o el Caribe (Wardle, 2007). Aunque muchos de ellos se basan en un modelo de los procesos tempranos de globalización — conocidos como protoglobalización, globalización arcaica o primera globalización-, son relevantes para el cosmopolitismo, porque cambian los términos del análisis histórico y geográfico, por ejemplo, al cuestionar las categorías de centro y periferia, universalismo y diferencia, local y global (véase también Hopkins, 2006). Han tenido un impacto significativo en el pensamiento histórico de la China como una alternativa a las concepciones eurocéntricas de la historia (Liu, 2011).

Sin embargo, cuando se invoca el cosmopolitismo es en gran medida como una categoría vaga para referirse a los intercambios transculturales principalmente en contextos premodernos y no posee una importancia normativa clara. El intercambio y la movilidad cultural en sí no deberían equipararse a la condición de cosmopolitismo. Son más bien importantes precondiciones que también pueden producir resultados no cosmopolitas (véase Delanty, 2011). Por este motivo, existen ciertos límites a lo que el giro de la historia global puede ofrecer al cosmopolitismo en la medida en que este último implica aspectos normativos. Los desarrollos recientes en la historia intelectual global demuestran que es difícil minimizar la arrolladora importancia de la difusión global del pensamiento occidental, por mucho que las tradiciones no occidentales hayan producido alternativas o reinterpretaciones (véase, por ejemplo, Moyn y Sartori, 2013).

9. Pueden encontrarse muchos ejemplos históricos de culturas cosmopolitas en el Journal of World History. El volumen 24 (3) es un número especial sobre aproximaciones históricas al cosmopolitismo. 


\section{Universalismo cognitivo}

No hay ninguna razón por la que las aproximaciones universalistas deban confinarse a las religiones mundiales o a las tradiciones indígenas o precoloniales. $\mathrm{El}$ argumento de que la modernidad ofrece un marco general para el mundo es también una forma de ver cómo es posible relacionarnos con lo que tenemos en común. Puede denominárselo "universalismo cognitivo» para indicar que el contenido universalista concierne a los principios de la razón que no son específicos de una única cultura, sino que más bien residen en las estructuras de la consciencia que se asocian a la modernidad, como la idea de la autonomía humana, la libertad, la igualdad y el valor moral del individuo. Debe distinguirse del universalismo moral, que simplemente mantiene que existen valores o principios morales que trascienden la cultura y que pueden ser la base de un orden cosmopolita de valores ${ }^{10}$. El universalismo cognitivo consideraría que estos valores están arraigados en todas las culturas en grados distintos, pero no vería factible que los individuos simplemente pudieran trascender sus culturas. Mientras que las diferentes culturas han interpretado de maneras diversas estas «ideas de la razón», ellas no son el producto de un solo país o civilización. El universalismo cognitivo es, por lo tanto, un tipo mínimo de cosmopolitismo (aunque no tan mínimo como el universalismo moral) y, potencialmente, una base para superar no sólo el eurocentrismo, sino también todas las aproximaciones etnocéntricas. En esta visión, que está cerca de la posición justificatoria, existen ideas centrales que son constitutivas de todas las culturas, incluso cuando algunas las han realizado más plenamente que otras. Por consiguiente, ciertos valores que a menudo se consideran europeos - democracia, libertad, autonomía, etc.- no son específicos de Europa, sino que, simplemente, se desarrollaron antes en ella. Dependiendo de cómo estos valores se traduzcan conceptual y culturalmente, el problema del eurocentrismo podría desaparecer como resultado de la contextualización global.

Una ilustración de la dimensión cognitiva respecto al cosmopolitismo es reemplazar dicho término por otro que pueda traducirse más fácilmente a contextos culturales distintos. Por ejemplo, un candidato para ello es la noción de unidad en la diversidad, un término que tiene ecos en el pensamiento europeo, así como en el indio y en el chino. Hans Georg Gadamer (1992) mantenía, en "La diversidad de Europa», que la herencia de Europa es la unidad en la diversidad: «Esta unidad debe extenderse al mundo entero, incluyendo Japón, China, la India y también las culturas musulmanas. Cada cultura, cada pueblo tiene algo distintivo que ofrecer para la solidaridad y el bienestar de la humanidad» (citado en Dallmayr, 1994: XIII). Mientras que el modelo teórico de Gadamer estaba más próximo a la noción de un diálogo de civilizaciones, la idea de un principio más amplio que se refleja en civilizaciones distintas puede verse como un principio cognitivo transcultural. El diálogo es una base importante del cosmopolitismo, como se ha apuntado antes, pero también requiere a menudo

10. Recurro al concepto de cognitivo de Strydom (2011, 2012). 
la traducción para hacerlo inteligible. La noción de unidad en la diversidad posee hasta cierto punto una función transcultural y traduce mucho de la idea de cosmopolitismo; por ejemplo: podría verse como una traducción de la noción china de tianxia, que, generalmente, se traduce por 'todos bajo el cielo' (véase He y Brown, 2012; Rofel, 2012). Este es probablemente el equivalente más cercano al cosmopolitismo en el discurso político chino contemporáneo ${ }^{11}$. El cosmopolitismo definido como 'la unidad en la diversidad' puede relacionarse fácilmente con el pensamiento indio (véase Padmanabhan, 2012). Es un concepto arraigado en la constitución de la India y forma parte de la identidad de la Unión Europea (Oommen, 2004). La noción japonesa de Kyosei también traduce el cosmopolitismo para incluir la idea de la participación en una comunidad global, la superación del prejuicio y el reconocimiento de la diferencia (Sugimoto, 2012).

No obstante, yo aduciría que un concepto traducible no es en sí mismo suficiente, pues debe hacerse algo con el concepto traducido que se adecue a la tarea de abordar las cuestiones globales contemporáneas. La noción de unidad en la diversidad quizás sea un recurso, pero no es necesariamente suficiente en sí misma. Lo que se necesita es un impulso normativo y crítico más fuerte. Por estas razones, la traducción cultural es insuficiente para un marco de análisis cosmopolita, a no ser que venga acompañada de un impulso normativo y crítico más fuerte. Esto me lleva al cosmopolitismo crítico.

\section{Cosmopolitismo crítico}

Dentro de esta amplia categoría, tal como se la usa aquí, el problema del eurocentrismo es sólo un aspecto y no la preocupación que la define. El cosmopolitismo crítico difiere de los tres primeros enfoques antes mencionados en su actitud más crítica hacia las tradiciones culturales, tanto europeas como no europeas, y en que preserva una posición normativa que no descarta la posibilidad de la trascendencia cultural. Es también en este aspecto que se distingue en cierta medida de otros usos del término, que se refieren más al problema de la diferencia cultural (Mignolo, 2000b; véase también Rabinow, 1986). Por su propia naturaleza, el cosmopolitismo es crítico en su autocomprensión básica, pero esto no está siempre muy marcado en la mayor parte de las principales versiones, como las discutidas anteriormente o en la teoría política normativa. El cosmopolitismo crítico enfatiza la naturaleza abierta de los encuentros entre las culturas y que las culturas aprenden unas de otras; no meramente que se producen tales encuentros (Delanty, 2009, 2014). No se trata, por lo tanto, simplemente de encontrar en las culturas del mundo valores comunes u ocasiones de intercambio y pluralidad, sino de identificar fuentes para el diálogo crítico y de cultivar el pensamiento crítico. Se construye sobre

11. Puede objetarse que tianxia se limitaba generalmente al Reino medio. Aunque sea cierto, también podría decirse que el término griego original, kosmopolitês, se limitaba al Imperio alejandrino, y el orden cosmopolita de Kant, al sistema europeo de estados. 
la idea del universalismo cognitivo, pero lo trasciende al integrar las instancias de cosmopolitismo a contextos específicos de justificación. Desde la perspectiva del cosmopolitismo crítico, el factor clave es el desarrollo de poderes críticos que cuestionen una visión estrecha del mundo. Sin embargo, a diferencia de las posiciones universalistas fuertes, el sujeto cosmopolita siempre está arraigado a un contexto cultural determinado. La imaginación cosmopolita es la capacidad para pensar más allá de este contexto a la luz de principios universalistas. Para desarrollar esta idea, tres posibles estrategias metodológicas ilustran un amplio enfoque cosmopolita crítico en las ciencias sociales.

Mientras que el cosmopolitismo europeo dominante se ocupaba casi exclusivamente del contexto interno europeo, un enfoque alternativo más crítico buscaría identificar en la cultura europea, incluida la tradición cosmopolita, la influencia positiva de ideas no europeas. Contrariamente a la crítica orientalista de Europa, muchos pensadores de la Ilustración (como Schlegel o Leibniz) eran muy conscientes de la contribución positiva de otras partes del mundo, especialmente de Asia. Alexander von Humboldt, por ejemplo, poseía un sentido inclusivo del mundo animado por una enérgica crítica de la esclavitud. Influido por el romanticismo, desarrolló un tipo de compromiso que trascendía los supuestos liberales de muchos pensadores de la Ilustración. Ahí se encuentra, por lo tanto, una de las estrategias para un cosmopolitismo crítico poseurocéntrico: el descentramiento de Europa a través del descubrimiento de lo no europeo dentro de Europa. Este enfoque, que podría describirse como una Europa que descubre su propia otredad interior, incluiría el análisis de las dimensiones críticas del pensamiento europeo que se oponían a una visión estrechamente eurocéntrica del mundo y buscaban cultivar una consciencia mundial en la que pudieran figurar otras culturas en términos de igualdad. La cuestión está en descubrir cómo el cambio en una parte del mundo impacta en otras partes, lo cual produce niveles más altos de reflexividad, por ejemplo, para dilucidar cómo los encuentros con el mundo no occidental influyeron en la Ilustración europea de una manera que la crítica orientalista o poscolonial no puede explicar (véase Clarke, 1997; Dallmayr, 1996).

Una segunda vía de investigación es el análisis sobre cómo las culturas no europeas se apropiaron de la cultura occidental sin aceptarla en su totalidad. Los enfoques principales dan por sentada la importancia global del pensamiento occidental sin considerar las formas en las que éste ha sido apropiado de maneras creativas, lo cual, en muchos casos, ha producido resultados híbridos. Esto puede adoptar formas muy diversas a través de las que ideologías occidentales como el socialismo o el positivismo, pongamos por caso, fueron trasplantadas a entornos muy distintos de su contexto original europeo, por ejemplo: el socialismo y el positivismo en la China y en Brasil, respectivamente. En otros casos (un ejemplo pertinente es Japón), las ideas occidentales fueron importadas paulatinamente a través de préstamos, reinterpretaciones y de su absorción en tradiciones nativas (Delanty, 2003). También supondría examinar la relevancia de conceptos como los de sociedad civil, democracia o esfera pública para la historia islámica, por ejemplo (véase Browers, 2006; véase tam- 
bién Chambers y Kymlicka, 2002), o considerar que el confucionismo posee en su interior una tradición democrática característica que emana de él a través de la crítica inmanente, en oposición a la simple adopción de formas occidentales (Ackerly, 2005). Desde la perspectiva del cosmopolitismo crítico, en oposición al cosmopolitismo contracivilizatorio o alternativo discutido más arriba, el propósito no sería necesariamente la identificación de una alternativa nativa a las categorías occidentales, sino una perspectiva que pretende identificar los potenciales transformadores de ambas culturas, la occidental y la oriental. Parte de esta tarea crítica es ver cómo puede reinterpretarse el pasado con categorías no eurocéntricas. Por ejemplo, el descubrimiento de la Carta del Mandén del siglo XIII en la cuenca alta del Níger cuestiona nuestras nociones recibidas de la historia africana y demuestra que existía en África una tradición constitucional preoccidental ${ }^{12}$. Puede demostrarse que la construcción orientalista de la ciudadanía como una práctica exclusivamente occidental es artificial, como lo revelan Isin y Lefrebvre (2005) respecto a la práctica de obsequiar en las instituciones del evergetismo griego y del waaf otomano.

Una tercera estrategia incluiría la exploración de la importancia global de conceptos no europeos, como cara y guanxi, tal como ha mantenido Xiaoying Qi (Qi, 2011, 2012, 2013). Es algo relevante para la tarea de superar el privilegio eurocéntrico de conceptos occidentales, aunque normalmente no se lo aborde desde la perspectiva del cosmopolitismo.

Todas estas estrategias implican en grados diversos no sólo la traducción conceptual, sino también la traducción cultural en una dirección que captura el momento normativo y crítico de autotransformación y aprendizaje a la luz del encuentro con el otro. Demuestran que las culturas, a pesar de sus diferencias, no son completamente inconmensurables. Ello no se debe a la existencia de valores comunes en sí, sino que puede atribuirse a la existencia de recursos cognitivos comunes capaces de conducir a una crítica cultural inmanente, por lo que ofrecería una respuesta al problema de cómo decidir qué tradiciones son relevantes para el cosmopolitismo, puesto que no todas lo son y pueden ser mutuamente contradictorias además de internamente diversas, como, por ejemplo, la mezcla en la civilización india entre hinduismo, budismo y jainismo, y en la China entre el daoísmo y el confucianismo. El islam y el cristianismo también manifiestan numerosas fracturas. Y el mismo cosmopolitismo occidental se compone de muchas corrientes distintas (los cínicos versus los estoicos, la tradición kantiana, el cosmopolitismo de Marx y las disposiciones humboldtianas (Walls, 2009)).

La noción de hermenéutica diatópica, en propuestas diversas de Raimon Panikkar y Boaventura de Sousa Santos (2007), es relevante para demostrar lo que Santos ha identificado como lo «incompleto» de todas las perspectivas culturales y, por lo tanto, contribuye a la solución de este problema. Esta visión sugiere que una hermenéutica cosmopolita adecuada requiere la capacidad para ver el mundo desde una variedad de perspectivas distintas y

12. Véase http://www.unesco.org/culture/ich/index.php?lg=es\&pg=00011\&RL=00290. 
no desde una sola. Sin embargo, no responde a la pregunta sobre qué tipo de cosmopolítica resultará de ello, puesto que subsiste una cuestión clave: la de si conseguir una perspectiva múltiple es suficiente para solucionar los principales problemas globales. Más bien podría ser considerado como un paso fundamental en la dirección de una cosmopolítica, pero no puede ser el resultado final. Es más, la posición cosmopolita crítica que he planteado pone un marcado acento en la posibilidad de evaluar críticamente los puntos de vista culturales.

Por lo tanto, lo que se propone es más bien un tipo normativo de cosmopolitismo crítico que supere la particularidad de las tradiciones culturales, sin necesariamente trascenderlas. El cosmopolitismo también es un proyecto político que apunta a la superación de las diferencias divisivas y no sólo una condición de entendimiento intercultural derivado de las concepciones del mundo o las herencias culturales de las religiones de la era axial. Como proyecto cosmopolítico, va más allá del problema del eurocentrismo, que es a menudo demasiado prominente en las nuevas aproximaciones al cosmopolitismo, para explorar las posibilidades alternativas del presente. Una de las características importantes del cosmopolitismo hoy es la visión alternativa que ofrece a la globalización y al nacionalismo. El cosmopolitismo es una crítica, tanto de una visión del mundo basada en naciones cerradas, como de la visión de un mundo homogeneizado por la globalización. Por lo tanto, el reconocimiento de las variedades mundiales de cosmopolitismo no debería conducir a la conclusión de la inconmensurabilidad. Civilizaciones distintas pueden, sin duda, poseer tradiciones cosmopolitas distintas, cuya comprensión es ciertamente importante para la política cosmopolita y es posible traducir entre ellas sólo por el hecho de que todas se han influido mutuamente. No existe ninguna tradición que se defina por un significado original que haya permanecido constante. En el caso de la tradición europea de cosmopolitismo, como he mantenido, ha experimentado diversos cambios de significado, hasta el punto de que es difícil hablar de una tradición continua. Creo que sucede lo mismo con otras tradiciones y que todas ellas han sido influidas por siglos de enriquecimiento mutuo.

\section{Conclusión}

En un último análisis, uno de los candidatos más prometedores para una exitosa traducción cultural es la noción de «hospitalidad», que Kant promulgaba en sus escritos políticos ${ }^{13}$, pero que parece poseer un amplio eco contemporáneo en todas las culturas del mundo y que no es específica a su contexto de origen. Este principio demanda reconocer que no debe tratarse a los seres humanos con hostilidad, sino con hospitalidad, estén donde estén. Numerosos ejemplos demuestran que las ideas, los valores y los principios culturales pueden trascender su contexto de origen, como la difusión más o menos universal 
del concepto de nación y, más recientemente, de los derechos humanos. El principio de la hospitalidad -más que las nociones de unidad y diversidad, de humanidad común o de diálogo de civilizaciones - captura el centro substantivo del cosmopolitismo de una forma que facilita la traducción cultural en todos los contextos civilizatorios. No creo que se trate principalmente de una idea europea, e incluso aunque fuera articulada antes en Europa ha perdido ya su especificidad europea.

Para concluir, el campo del cosmopolitismo es muy vasto y, cuando se extiende para abarcar a las tradiciones no occidentales, el conjunto potencial de temas se amplía aún más. He intentado acentuar algunas cuestiones normativas y metodológicas clave que son relevantes para superar los prejuicios eurocéntricos que, por otra parte, he advertido que no conviene exagerar, sin caer en posiciones ni contextualistas extremas ni universalistas. En mi opinión, el cosmopolitismo ofrece un enfoque para la investigación comparada, al abordar lo que las sociedades tienen en común. No obstante, ello requerirá ir más allá de las presuposiciones del análisis meramente comparativo de sociedades separadas, puesto que debemos ahora asumir la naturaleza interconectada de las sociedades. El reconocimiento de esta interconectividad posibilita un grado de traducción cultural.

Los debates de la filosofía comparada y la historia global son, como he sostenido, muy importantes para formular nuevas preguntas, pero la teoría cosmopolita debe ir más allá de estas aproximaciones que, generalmente, se limitan al análisis histórico, para identificar posibilidades alternativas en el presente. No se trata sólo de identificar tradiciones culturales alternativas a las occidentales que puedan ser portadoras de distintos tipos de valores cosmopolitas, sino de identificar en estas tradiciones culturales distintas los recursos para una cosmopolítica. Esto sugiere, entonces, que lo que debemos buscar no es meramente un cosmopolitismo chino, o un cosmopolitismo japonés o indio, por ejemplo, como alternativas al cosmopolitismo europeo, sino ver cómo estas tradiciones, que en sí mismas son todas ellas incompletas, podrían ofrecer recursos para el presente. No obstante, el reconocimiento de las variedades mundiales de cosmopolitismo es un paso esencial en el desarrollo de un proyecto cosmopolítico.

El análisis de este artículo ha revelado que las corrientes principales no se involucran adecuadamente con las culturas no occidentales y sus tradiciones cosmopolitas, o que, cuando lo hacen, tienden a caer en posiciones liberales o poscoloniales. Las primeras tienden a ser demasiado mínimas y las segundas demasiado englobadoras, al ubicar el cosmopolitismo en los contradiscursos. Desde el punto de vista privilegiado del cosmopolitismo crítico, todos estos enfoques carecen tanto del objetivo hermenéutico de la traducción cultural como de la tarea crítica de ampliar los horizontes morales y políticos de la sociedad. 


\section{Referencias bibliográficas}

Ackerly, B. (2005). "Is liberalism the only way toward democracy?: Confucianism and democracy». Political Theory [en línea], 33 (4), 547-576. <http://dx.doi.org/10.1177/0090591705276879>.

Alatas, S. F. (2006). Alternative Discourses in Asian Social Science: Responses to Eurocentrism. Nueva Delhi: SAGE.

Angle, S. (2012). Sagehood: The Contemporary Significance of Neo-Confucian Philosophy. Oxford: Oxford University Press.

Beck, U. (2006). The Cosmopolitan Outlook. Cambridge: Polity Press.

Bell, D. (2000). East Meets West: Human Rights and Democracy in Asia. Princeton: Princeton University Press.

- (2006). Beyond Liberal Democracy: Political Thinking for an East Asian Context. Princeton: Princeton University Press.

Benhabib, S. (2011). Dignity in Adversity: Human Rights in Troubled Times. Cambridge: Polity Press.

Benjamin, W. (1970). «The task of the translator». En: Illuminations. Londres: Fontana.

Breckenridge, C. A.; Bhabha, H.; Pollock, H. y Chakrabarty, D. (eds.) (2002). Cosmopolitanism. Durham, NC: Durham University Press.

Brock, G. (2009). Global Justice: A Cosmopolitan Account. Oxford: Oxford University Press.

Browers, M. (2006). Democracy and Civil Society in Arab Political Thought: Transcultural Possibilities. Syracuse, NY: Syracuse University Press.

Burik, S. (2009). The End of Comparative Philosophy and the Task of Comparative Thinking. Nueva York: State University of New York Press.

Chakrabarty, D. (2000). Provincializing Europe: Postcolonial Thought and Historical Difference. Princeton: Princeton University Press.

Chambers, S. y Kymlicka, W. (eds.) (2002). Alternative Conceptions of Civil Society. Princeton: Princeton University Press.

Cheah, P. (2006). Inhuman Conditions: On Cosmopolitanisms and Human Rights. Cambridge, MA: Harvard University Press.

Clarke, J. (1997). Oriental Enlightenment: The Encounter between Asian and European Thought. Londres: Routledge.

Connell, R. (2006). "Northern theory: The political geography of general social theory». Theory and Society [en línea], 35, 237-264. $<$ http://dx.doi.org/10.1007/s11186-006-9004-y>.

Conrad, S. (2012). "Enlightenment in global history: A historiographical critique». American Historical Review [en línea], 117 (4), 998-1027. <http://dx.doi.org/10.1093/ahr/117.4.999>.

Dallmayr, F. (1996). Beyond Orientalism: Essays on Cross-Cultural Encounter. Nueva York: State University of New York Press.

Delanty, G. (2003). "Japan and modernity». En: Beck, U.; Sznaider, N. y Winter, R. (eds.). Global America: The Cultural Consequences of Globalization. Liverpool: Liverpool University Press.

- (2009). The Cosmopolitan Imagination: The Renewal of Critical Social Theory. Cambridge: Cambridge University Press.

- (2011). "Cultural diversity, democracy and the prospects of cosmopolitanism: A theory of cultural encounters». British Journal of Sociology [en línea], 62 (4), 633-656. <http://dx.doi.org/10.1111/j.1468-4446.2011.01384.x>. 
- (ed.) (2012). The Handbook of Cosmopolitanism Studies. Londres: Routledge.

- (2014). "The prospects of cosmopolitanism and the possibility of global justice». Journal of Sociology [en línea], 50 (2), 213-228. <http://dx.doi.org/10.1177/1440783313508478>.

Euben, R. (2008). Journeys to the Other Shore: Muslims and Western Travelers in Search of Knowledge. Princeton: Princeton University Press.

GADAmER, H.-G. (1992). "The diversity of Europe: Inheritance and future». En: MisGeld, D. y Nicholson, G. (eds.). Applied Hermeneutics. Nueva York: SUNY Press.

Getz, T. (2013). Cosmopolitan Africa, c 1700-1875. Oxford: Oxford University Press.

Go, J. (2013a). «For a postcolonial sociology». Theory and Society, 42, 25-55.

- (2013b). «Fanon's postcolonial cosmopolitanism». European Journal of Social Theory, 16 (2), 208-225.

Godrej, F. (2011). Cosmopolitan Political Thought. Londres: Routledge.

Gray, S. (2010). "A historical comparative approach to Indian political thought: Locating and examining domesticated differences». History of Political Thought, 31 (3), 383-406.

Hawley, J. (ed.) (2008). India in Africa, Africa in India: Indian Ocean Cosmopolitanism. Indianápolis: Indiana University Press.

He, B. y Brown, K. (2012). "An empirical world of Asian cosmopolitanism». En: Delanty, G. (ed.). Routledge Handbook of Cosmopolitanism Studies. Londres: Routledge.

Holton, R. (2009). Cosmopolitanisms: New Thinking and New Directions. Londres: Palgrave.

Hopkins, A. G. (ed.) (2006). Interactions between the Universal and the Local. Londres: Palgrave.

Isin, E. y Lefebvre, A. (2005). "Greek euergetism and Ottoman waqf». European Journal of Social Theory [en línea], 8 (5), 5-23. <http://dx.doi.org/10.1177/1368431005046707>.

Jenco, L. (2007). “"What does heaven ever say?”: A methods-centred approach to cross-cultural engagement». American Review of Political Science, 101 (4), 741-55.

- (2012). «How meaning moves: Tan Sitong on borrowing across cultures». Philosophy East and West [en línea], 62 (1), 92-113. $<$ http://dx.doi.org/10.1353/pew.2012.0007>.

Kleingeld, P. (1999). «Six varieties of cosmopolitanism». Journal of the History of Ideas [en línea], 60 (3), 505-24. <http://dx.doi.org/10.1353/jhi.1999.0025>.

LIU, X. (2011). "A Silk Road legacy: The spread of Buddhism and Islam». Journal of World History [en línea], 22 (1), 55-81. $<$ http://dx.doi.org/10.1353/jwh.2011.0021>.

Lukes, S. (2000). «Different cultures, different rationalities?». History of the Human Sciences, 13 (1), 5-18.

Meijer, R. (ed.) (1999). Cosmopolitanism, Identity and Authenticity in the Middle East. Londres: Curzon Press.

Mignolo, W. (2000a). Local Histories/Global Designs. Princeton: Princeton University Press.

- (2000b). «The many faces of cosmo-polis: Border thinking and critical cosmopolitanism». En: Breckenridge, C. A. et al. (eds.). Cosmopolitanism. Durham, NC: Durham University Press. 
Мота, A. (2012). «Cosmopolitanism in Latin America: Political practices, critiques and imaginaries». En: Delanty, G. (ed.). Routledge Handbook of Cosmopolitanism Studies. Londres: Routledge.

Moyn, S. y SARTOri, A. (eds.) (2013). Global Intellectual History. Nueva York: Columbia University Press.

Oommen, T. K. (2004). "Socio-political transition in the Indian Republic and the European Union». European Journal of Social Theory [en línea], 7 (4), 519-37. <http://dx.doi.org/10.1177/1368431004046706>.

Padmanabhan, S. (2012). "Unity in diversity: The Indian cosmopolitan idea». En: Delanty, G. (ed.). Routledge Handbook of Cosmopolitanism Studies. Londres: Routledge.

Pollock, S. (2006). The Language of the Gods in the World of Men: Sanskrit, Culture, and Power in Premodern India. Berkeley: University of California Press.

Pomeranz, K. (2000). The Great Divergence: China, Europe and the Making of the World Economy. Princeton: Princeton University Press.

QI, X. (2011). "Face: A Chinese concept in a global sociology». Journal of Sociology, 47 (3), 279-296.

- (2012). «A case study of globalized knowledge flow: Guanxi in social science and management theory». International Sociology [en línea], 27 (6), 707-723. <http://dx.doi.org/10.1177/0268580912453729>.

- (2013). "Guanxi, social capital theory and beyond: Towards a global social science». British Journal of Sociology [en línea], 64 (2), 308-22. $<$ http://dx.doi.org/10.1111/1468-4446.12019>.

Rabinow, P. (1986). «Representations are also social facts». En: Clifford, C. (ed.). Writing Culture: The Poetics and Politics of Ethnography. Berkeley: University of California Press.

Rendall, G.; Skrbis, Z. y Woodward, I. (2009). The Sociology of Cosmopolitanism. Londres: Palgrave.

Rofel, L. (2012). «Between Tianxia and postsocialism: Contemporary Chinese cosmopolitanism». En: Delanty, G. (ed.). Routledge Handbook of Cosmopolitanism Studies. Londres: Routledge.

SAntos, B. (2007). "Human rights as an emancipatory script». En: SANTos, B. (ed.). Another Knowledge is Possible: Beyond Northern Epistemologies. Londres: Verso.

Sheriff, A. (2010). The Dhow Culture of the Indian Ocean: Cosmopolitanism, Commerce and Islam. Nueva York: Columbia University Press.

Sitas, A. (2006). "The African Renaissance challenge and sociological reclamations in the South». Current Sociology [en línea], 54 (3), 357-80. <http://dx.doi.org/10.1177/0011392106063186>.

Skrbis, Z. y Woodward, I. (2013). Cosmopolitanism: Uses of an Idea. Londres: SAGE.

STRYDOM, P. (2011). "The cognitive and metacognitive dimensions of contemporary social and political theory». En: Delanty, G. y Turner, S. (eds.). International Handbook of Contemporary Social and Political Theory. Londres: Routledge.

- (2012). «Modernity and cosmopolitanism: From a critical social theory perspective». En: Delanty, G. (ed.). Handbook of Cosmopolitanism Studies. Londres: Routledge.

Sugimoto, Y. (2012). «Kyosei: Japan's cosmopolitanism». En: Delanty, G. (ed.). Routledge Handbook of Cosmopolitanism Studies. Londres: Routledge.

WALlerstein, I. (1997). «Eurocentrism and its avatars: The dilemmas of social science». New Left Review, 1 (226), 93-107. 
Walls, D. L. (2009). The Passage to Cosmos: Alexander von Humboldt and the Shaping of America. Chicago: University of Chicago Press.

WANG, H. (2012). "Ren and Gantong: Openness of heart and the root of Confucianism». Philosophy East and West [en línea], 62 (4), 463-4. <http://dx.doi.org/10.1353/pew.2012.0067>.

WARDLE, H. (2007). "A groundwork for West Indian cultural openness». Journal of the Royal Anthropological Institute [en línea], 13, 567-83. <http://dx.doi.org/10.1111/j.1467-9655.2007.00444.x>.

Werbner, P. (ed.) (2008). Anthropology and the New Cosmopolitanism. Oxford: Berg. Xincheng, L. (2012). "The global view of history in China». Journal of World History [en línea], 23 (4), 491-511. <http://dx.doi.org/10.1353/jwh.2012.0084>. 